ISSUES IN PUBLIC HEALTH

\title{
When do adolescent mothers return to school? Timing across rural and urban South Africa
}

\author{
J Jochim, ${ }^{1}$ BSc, MSc; A K Groves, ${ }^{2}$ BA, MHS, PhD; L D Cluver, ${ }^{1}$ MA, MSc, MScDipSW, PhD \\ ${ }^{1}$ Department of Social Policy and Intervention, Social Sciences Division, University of Oxford, UK \\ ${ }^{2}$ Department of Community Health and Prevention, Dornsife School of Public Health, Drexel University, Philadelphia, PA, USA
}

Corresponding author: Jochim (janina.jochim@spi.ox.ac.uk)

\begin{abstract}
While adolescent mothers' postpartum return to school offers long-term benefits, returning too early in the absence of sufficient support may impede the healthy development of their children. This study assessed the rates of adolescent mothers' return to school in South Africa (SA) and examined how many months after birth school-aged girls resumed schooling. Two independent studies recruited 1114 adolescent mothers in Eastern Cape Province and Durban, respectively. Across the two studies, 64.7\% ( $n=649)$ and $47.8 \%(n=53)$ of mothers, respectively, had returned to school at the time of the study. Of these mothers, the majority had returned to school within the first 2 months post partum (study 1: 67.6\%; $n=439$; and study 2: 58.5\%; $n=31$ ), fewer between 2 and 6 months $(16.9 \%$; $n=110$; and $22.6 \%$; $n=12$ ), and after 12 months $(2.0 \% ; n=13$; and unknown). Our findings indicate a mismatch between SA's national policy recommendations and actual return patterns, showing that a large proportion of mothers returned to school much earlier than advised. This study also highlights a particular need to amend school policies that support early-returners and their children. Additional research on the needs of mother-child dyads and studies on the impact of different timescales on educational and health outcomes are needed to further inform policy and practice regarding adolescent mothers' return to school.
\end{abstract}

S Afr Med J 2020;110(9):850-854. https://doi.org/10.7196/SAMJ.2020.v110i9.14664

In South Africa (SA), 125 000 girls aged 10 - 19 years experience a pregnancy every year. ${ }^{[1]}$ Rates of adolescent pregnancy are much higher in populations affected by extreme adversities, which limit their educational attainment and specifically their graduation from high school. ${ }^{[2-5]}$ Previous studies found that only $30-50 \%$ of girls return to school after birth or a pregnancy-related dropout. ${ }^{[6,7]}$ Evidence shows that the longer a mother stays out of school after giving birth, the less likely she is to return. ${ }^{[7]}$

While SA's 2007 national policy ('Measures for the prevention and management of learner pregnancy') stipulates that learners are allowed to stay in school while pregnant, the policy also recommends that mothers should not return to school in the same year during which the pregnancy occurred. The policy specifies that young parents may take leave for up to 2 years to exercise full parenting responsibility, and requires the girls to provide a medical report that declares the learner fit for school upon their return. ${ }^{[8]}$ In 2009, the Human Sciences Research Council published a report on behalf of the Department of Education (DoE), which criticised these stipulations and called for revisions. These included suggestions for flexible school policies that enable early re-entry of young mothers into the schooling system. ${ }^{[9]}$ Despite these early suggestions, no official changes to the national policy have been made.

A small number of empirical studies provided valuable insights into the rates of postpartum schooling of adolescent mothers. Specifically, two longitudinal studies showed that between 35\% and $50 \%$ of girls who had a child before completing high school were enrolled in school during the year after birth. ${ }^{[6,10]}$ Examining how schooling further unfolds over the years after birth, one study indicated that there is an ongoing decrease in enrolment rates after the first year post partum, showing that an increasing number of girls drop out of school as time passes. ${ }^{[6]}$ By contrast, other studies suggested that the percentage of girls that are enrolled in school is upward sloping after the first year post partum. This provides some evidence that a few adolescent mothers who had dropped out do return to school at some point after giving birth. ${ }^{[10,11]}$

While the abovementioned longitudinal studies ${ }^{[6,10,11]}$ report rates of return to school in the first year after birth, there is no research on the specific timing of school return. We have no data on how many mothers returned in the first month after birth v. how many returned when their baby was closer to 1 year old. These differences are meaningful to the health and wellbeing of the mother and child and warrant further attention. Assessing when adolescent mothers return to school after the birth of their child is also important for the development of school policies that effectively support mother and child needs by recommending realistic timings for school return.

The purpose of our article is to present novel data from two independent projects on the timing of adolescent mothers' school return across two SA provinces. The findings are presented with reference to the recommended timings outlined in SA's current national policy, and their relevance for the development of future policies are discussed.

\section{Methods}

\section{Study 1}

Study 1 was the first wave of a prospective cohort study conducted in rural and semiurban areas of Eastern Cape Province, SA. Between 2017 and 2019, 1003 adolescent mothers (aged 12 - 19 years) were recruited by healthcare facilities $(40 \%)$, community-based strategies (26\%), schools (10\%), peer referrals (6.5\%), non-governmental organisations (NGOs) (4\%) and from a cohort of adolescents, with whom the research team had previously worked (13\%).

Informed consent was sought from adolescents who were $>18$ years old, while caregivers provided consent for underage participants. Adolescent mothers completed two complementary self-report 
interviews, which asked a range of questions regarding their health, family, relationships, violence experiences and schooling. Each interview was undertaken by interviewers trained in working with vulnerable youth and lasted $\sim 60$ minutes.

There was no fixed time point post partum at which participants completed the interview (i.e. participants' children were aged between 3 months and 9 years). This meant that at the time of the interview, some mothers had had less time to return to school than others. All mothers were interviewed in private spaces in and around their own home, but they were given the option to conduct the interview in a local restaurant if the privacy in their home was compromised. Confidentiality was maintained throughout the study, except where participants requested help or were at risk of significant harm. In these cases, referrals were made to health or counselling services, with follow-up support. There were no monetary incentives, but all participants received a certificate, refreshments and a participant pack containing useful items, e.g. washcloth and soap.

\section{Ethical approval}

All study activities were approved by the institutional ethics boards at the University of Oxford (ref. nos R48876/RE001 and R48876/RE002) and the University of Cape Town (ref. no. 226/2017).

\section{Study 2}

Study 2 was a pilot intervention study, i.e. mentoring adolescent mothers at school (MAMAS), conducted in a periurban area of KwaZulu-Natal Province, SA. MAMAS was a non-equivalent comparison group study designed to support adolescent mothers in their return to school after childbirth. A total of 111 adolescent mothers (aged 14 - 19 years) from Umlazi township, who were part of the comparison group (i.e. they did not receive the MAMAS intervention), were recruited at a public maternity ward and at public health clinics between July 2017 and April 2018.

Participants completed a survey at $\sim 6$ months post partum, during which they answered questions on school experiences during pregnancy, returning to school after childbirth and timing of school return. Voluntary informed consent was obtained from adolescents and their caregivers in cases where adolescent mothers were underage. All interviews were completed on a tablet using audio-assisted computer interviewing. A trained research assistant was available to provide support to complete the surveys, as needed.

\section{Ethical approval}

All study activities were approved by the institutional ethics boards at Drexel University (ref. no. IRB:1612005048) and the University of KwaZulu-Natal (ref. no. BFC023/17).

\section{Resullts \\ Study 1}

This study was completed by 1003 participants. The mean age of study participants was 18.21 (standard deviation (SD) 1.80) years. Mothers in the sample were affected by several vulnerabilities (Table 1) - 26.6\% indicated not having enough food in the household in the past week and $92.7 \%$ came from families receiving at least one grant (mean 3.4; SD 2.1). The majority of mothers had only 1 child ( $n=916 ; 91.3 \%)$, whereas 87 mothers had $\geq 2$ children $(8.7 \%)$. The children were aged between 3 months and 9 years.

The majority of the 1003 mothers in the sample were enrolled in school when they fell pregnant with their oldest child $(n=902)$. After the birth of their oldest child, $64.7 \%(n=649)$ of mothers had returned to school, while $35.3 \%(n=354)$ had not returned to school at the time of the interview (Table 2). The median postpartum time to return was 1 (interquartile range (IQR) 0 - 2) month. Fig. 1(A) shows the return times of the 649 mothers who continued with school after childbirth. Most young mothers who had returned to school reported having returned $<1$ month after birth $(n=301)$.

\section{Study 2}

This study was completed by 111 participants. Their mean age was 17 (1.33) years, and the population was highly vulnerable (Table 1).

\section{Table 1. Sample characteristics}

\begin{tabular}{|c|c|c|c|}
\hline Group & Variable & Study $1(n=1003), n(\%)^{*}$ & Study $2(n=111), n(\%)^{*}$ \\
\hline \multirow[t]{8}{*}{ Sociodemographic characteristics } & Age, mean (SD) (months) & $18.21(1.80)$ & $17(1.33)$ \\
\hline & Rural residency & $293(29.2)$ & - \\
\hline & Food insecurity & 267 (26.6) (past week) & 59 (53.2) (past month) \\
\hline & Maternal orphan & $197(19.6)$ & $33(30.0)$ \\
\hline & Paternal orphan & $278(27.7)$ & $42(40.4)$ \\
\hline & Adults in the household, mean (SD) $(n)$ & $3.19(1.48)$ & $4.15(2.98)$ \\
\hline & Adolescent mother had $>1$ child & $87(8.7)$ & $7(6.48)$ \\
\hline & Family receives a grant & $930(92.7)$ & $88(79.3)$ \\
\hline \multirow[t]{3}{*}{ Educational characteristics } & Currently enrolled & $542(54.0)$ & Unknown \\
\hline & Highest completed grade, mean (SD) & $9.66(1.65)$ & Unknown \\
\hline & Repeated at least 1 grade & $577(60.9)$ & $35(31.6)$ \\
\hline
\end{tabular}

Table 2. Characteristics of school return

\begin{tabular}{lll}
\hline Variable & Study $\mathbf{1 , n = 1 ~ 0 0 3}$ & Study 2, $\boldsymbol{n = 1 1 1}$ \\
\hline Returned to school, $n(\%)$ & $649(64.7)$ & $53(47.8)$ \\
Returned to school, median (IQR) (months) & $1(0-2)$ & $1.25(0.72-4.11)$ \\
Returned to same school, $n(\%)$ & Unknown & $44(83.0)$ \\
Received help to return to school, $n(\%)$ & Unknown & $45(84.9)$ \\
SD = standard deviation; IQR = interquartile range. & &
\end{tabular}


Specifically, over half experienced food insecurity in the past month $(53.2 \%)$ and four-fifths were living in households that received at least one social grant (mean 1.45; SD 1.02). Furthermore, the majority were first-time mothers ( $n=104 ; 93.69 \%$ ).

With regard to schooling, just fewer than half of all participants had returned to school at the time of the interview $(n=53)$, which took place at $\sim 6$ months after childbirth (Table 2). Four-fifths of the girls returned to the same school and most reported that someone at home assisted them with the re-enrolment process. Fig. 1(B) shows the return times of the 53 mothers who continued school after childbirth. The median time to return was 1.25 (IQR 0.72 4.11) months. Four girls reported returning to school within 10 days of giving birth.

\section{Discussion}

The studies showed that $\sim 65 \%$ and $~ 50 \%$ of adolescent mothers from study 1 and 2 , respectively, had returned to school after giving birth. Overall, the presented rates are similar to those of previous studies, which reported that up to $50 \%$ of learners returned to school in the first year after giving birth, ${ }^{[6]}$ but higher than results from other studies, where only $30 \%$ returned. ${ }^{[7]}$ It is likely that the variability in the proportion of returned mothers across the 2 studies is partially due to the interviews being completed at different times post partum. Specifically, participants in study 2 completed interviews at $\sim 6$ months after birth and therefore had less time to return to school than participants in study 1 . Therefore, the higher number of returners in study 1 may be due to a number of girls who returned to school during the first few years after giving birth. ${ }^{[10,11]}$

Our results also indicated a mismatch between recommended timing for readmissions and actual return among SA adolescent mothers. A large proportion of mothers across both studies returned to school within the first 2 months after birth. Interestingly, this was the case across the 2 studies despite their differences in terms of study location, follow-up timing and sample size. The observed patterns indicate that school returns may occur much earlier than advised in SA's national policy on pregnant learners, which does not recommend returning to school in the same year that the pregnancy occurred. In line with previous studies, ${ }^{[7]}$ study 1 also showed that only a very small percentage of mothers returned 1 year after childbirth. This finding supports previous research commissioned by the DoE, which highlighted that the proposed 2-year leave

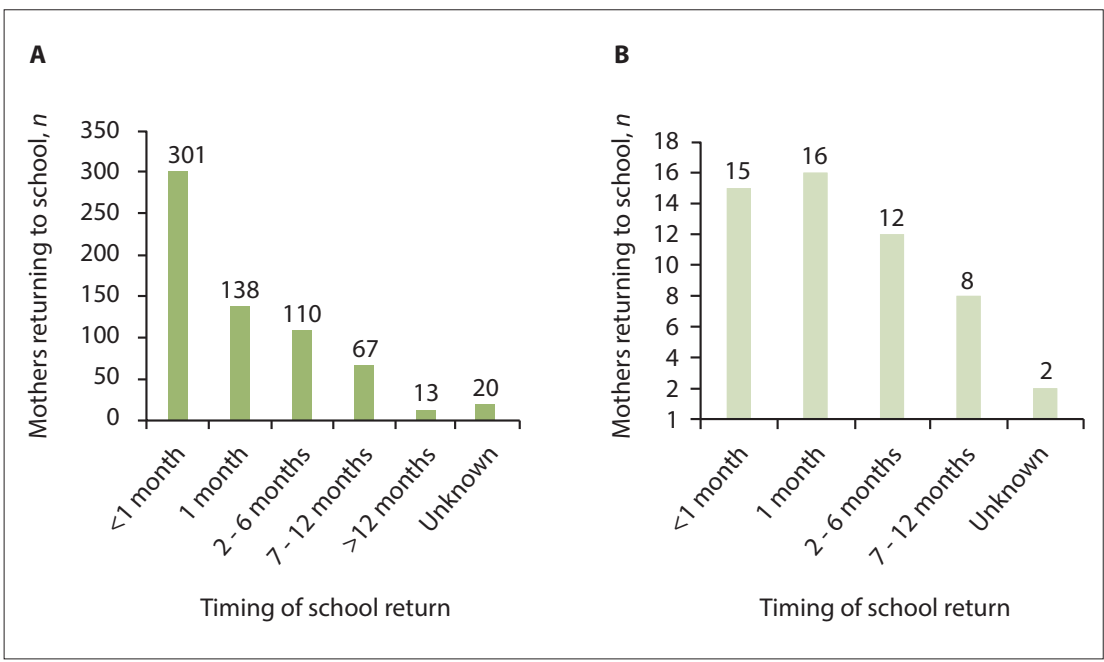

Fig. 1. Timing of school return for mothers who continued their education after giving birth. (A) Study 1 ( $\mathrm{n}=649) ;($ B) Study 2 ( $\mathrm{n}=53)$.

period may hinder mothers' readmission to school. ${ }^{[9]}$

Developing school policies that flexibly promote mothers' rights to education while simultaneously addressing the child's needs is difficult. SA's national policy may attempt to balance both goals, but our results indicate that the policy does not lead to uniform patterns of school return that follow the outlined recommendations. The high proportion of early returners in the 2 studies discussed above raises questions about if and how different schools are interpreting and implementing the policy in practice. It is possible that the policy is unknown or partly disregarded by some schools that perceive the recommended timings as ambiguous, overly restrictive or hard to implement. It is also possible that some schools follow the DoE-commissioned research and, as such, enable early re-entry for adolescent mothers. ${ }^{[9]}$ However, a government policy draft incorporating this research has not yet been made official. ${ }^{[12]}$

Our findings point to the particular urgency for policies that are flexible to the needs of adolescents who decide to return to school very soon after childbirth. The high prevalence of early returners in the current sample reinforces previous calls for policy amendments that provide mothers with the flexibility to return to school earlier than recommended in the national policy. ${ }^{[9]}$ Our results may indicate the level of demand for an updated policy that speaks to girls' realities. A refined policy that considers the timings of return identified in this study may contribute towards increased effectiveness and acceptability among adolescent mothers.

Recent research emphasised the importance of aligning educational policies for adolescent mothers with health policies that protect the needs and development of children. ${ }^{[13]}$ SA's Department of Health $(\mathrm{DoH})$ follows the World Health Organization (WHO) and the United Nations Children's Fund (UNICEF) recommendations ${ }^{[14]}$ and advises exclusive breastfeeding during the first 6 months, with gradual weaning, ${ }^{[15]}$ irrespective of HIV status. However, recent qualitative research involving $57 \mathrm{SA}$ adolescent mothers indicated low rates of exclusive breastfeeding, despite awareness of its benefits. Even though mothers reported various reasons for introducing mixed feeding early after birth, they indicated that schooling largely precludes exclusive breastfeeding. ${ }^{[13]}$

To ensure that policies affecting adolescent mothers are compatible with one another, the DoH and DoE should work on the development in unison. For instance, it would be intuitive for school policies to recommend a timeframe for school return that is aligned with the 6 months of recommended exclusive breastfeeding specified in the health policies. Maximising the health and wellbeing of mother-child dyads and acknowledging the nurturing needs of their children, require policies that not only address questions on school return timings but also outline ways in which the school context can actively support mothers after their return. Given the health benefits of breastfeeding, ${ }^{[16]}$ policy documents should make recommendations for the way in which schools can facilitate breastfeeding for schoolgoing adolescents who wish to breastfeed. The implementation of similar policies $^{[17]}$ that rely on collaborations between the DoH and DoE has proven somewhat challenging. ${ }^{[18-20]}$ Therefore, the success of 
an updated policy for adolescent mothers hinges on the political will and intersectional efforts to formulate clear, realistic and sustainable implementation processes.

For the development of successful school policies, it is also necessary that future research crystallises additional concrete needs and outcomes of schoolgoing mothers and their children. Building on past research that sheds light on how adolescent mothers navigate schooling, parental responsibilities and nurturing their child ${ }^{[13,21]}$ further studies should aim to elucidate the motivations and challenges experienced by early returners in particular. To develop social interventions aiming at reintegrating mothers into schools and at increasing breastfeeding uptake among schoolgoing mothers, it is important to identify the factors that contribute to mothers' decision-making in this context. Future research should aim to identify the different routes through which complementary policies from the DoH and DoE could address the factors associated with mixed feeding among adolescent mothers, ${ }^{[13]}$ which may interfere with school efforts to support schoolgoing mothers with exclusive breastfeeding. Lastly, the current findings highlight the need to assess if and how school policies targeting adolescent parents are monitored. Identifying the best ways to monitor the implementation of re-entry policies that promote the right to education is important to achieve educational equity for pregnant teenagers and adolescent mothers.

\section{Study limitations}

The 2 studies include several limitations. The cross-sectional nature of both studies precludes insights into the long-term, complex patterns of schooling behaviours. Neither study sought information on the schools' level of implementation on the current learner policy. This means that it is unclear whether or not the decision to re-enter school was guided by the national policy or the recommendations that emerged from research by the Human Sciences Research Council. Lastly, the 2 presented studies did not assess which breastfeeding practices were in place among the early-returning mothers. It is possible that mothers who returned to school very early decided against breastfeeding, were unaware that breastfeeding would provide benefits, were unable to breastfeed, were HIV-positive and advised not to breastfeed, or managed to breastfeed their child by means of various arrangements. Knowledge regarding the circumstances surrounding breastfeeding and other nurturing practices is needed to develop policies and programmes to support the realities of young mothers. Finally, our research was restricted to 2 provinces in SA. Further research with larger samples and in other provinces may increase the generalisability of the findings.

\section{Conclusions}

These are the first studies that report specific timings of return to school for SA adolescent mothers. Our findings highlight the complexity of developing school policies targeting adolescent mothers' return to school. School completion confers long-term benefits, ${ }^{[22-24]}$ but returning prematurely could potentially impair child development, ${ }^{[16]}$ and prolonged breaks can result in permanent dropout. To maximise the health and wellbeing of adolescent mothers and their children, future policies need to consider these consequences carefully and ensure that recommended timing reflects the best evidence-based practices.

\section{Declaration. None.}

Acknowledgements. We thank all adolescents who participated in this study, the respective field teams and support staff, and the University of Oxford and Drexel University for supporting this research. We also thank Ms Luwam Gebrekristos of Drexel University for her assistance as research co-ordinator for the Drexel University team.

Author contributions. AKG and LDC conceptualised each of the overarching studies and led data collection. AKG, JJ and LDC conceptualised the research question. AKG and JJ analysed the data. AKG and JJ compiled the tables. AKG, JJ and LDC interpreted the findings and wrote the paper.

Funding. The study by the University of Oxford and University of Cape Town was jointly funded by the UK Medical Research Council (MRC) and the UK Department for International Development (DFID) under the MRC/DFID Concordat agreement, and by the Department of Health and Social Care (DHSC) through its National Institutes of Health Research (NIHR) (MR/R022372/1); the European Research Council (ERC) under the European Union's Horizon 2020 research and innovation programme (no. 771468); the UK Research and Innovation and Global Challenges Research Fund (UKRI GCRF) Accelerating Achievement for Africa's Adolescents (Accelerate) Hub (grant ref. ES/S008101/1); the Fogarty International Center, National Institute on Mental Health, National Institutes of Health (award number K43TW011434) (the content is solely the responsibility of the authors and does not represent the official views of the National Institutes of Health); a Collaborative Initiative for Paediatric HIV Education and Research grant from the International AIDS Society (2018/625-TOS and 155-Hod) (the views expressed do not necessarily reflect the official policies of the International AIDS Society); and the Research England (0005218) and United Nations Children's Fund Eastern and Southern Africa Regional Office (UNICEF-ESARO). Additional co-funding was received from the Nuffield Foundation (CPF/41513) (the views expressed are those of the authors and not necessarily those of the Foundation); Evidence for HIV Prevention in Southern Africa (EHPSA), a UK aid programme managed by Mott MacDonald; Janssen Pharmaceutica NV, part of the Janssen Pharmaceutical Companies of Johnson \& Johnson and the Regional Inter-Agency Task Team for Children Affected by AIDS-Eastern and Southern Africa (RIATT-ESA). Further funding was provided by the Economic and Social Research Council (grant numbers ES/J500112/1; ES/R501037/1); the Leverhulme Trust (PLP-2014-095); the John Fell Fund; and the University of Oxford's Economic and Social Research Council Impact Acceleration Account. The data from the Drexel University study was funded by a grant from the US Department of State as part of the DREAMS Innovation Challenge, managed by the John Snow International Research and Training Institute, Inc (the opinions, findings, and conclusions stated herein are those of the authors and do not necessarily reflect those of the US Department of State or JSI). The development of this analysis was also supported by a Drexel Career Development award.

Conflicts of interest. None.

1. Maqhina M. Concern as more than 120000 schoolgirls fall pregnant. IOL News, 13 December 2019. https://www.iol.co.za/news/politics/concern-as-more-than-120-000-schoolgirls-fall-pregnant-39196455 (accessed 15 February 2020).

2. Moultrie TA, Timæus IM. The South African fertility decline: Evidence from two censuses and a demographic and health survey. Popul Stud 2003;57(3):265-283. https://doi.org/10.1080/00324720320 demographic and health survey. Popul Stud 2003;57(3):265-283. https://doi.org/10.1080/00324720320 00137808

3. Van Broekhuizen H, van der Berg S, Hofmeyr H. Higher education access and outcomes for the 2008 national matric cohort. Stellenbosch economic working papers. 2016. https://resep.sun.ac.za/wpcontent/uploads/2016/10/Van-Broekhuizen-et-al.pdf (accessed 15 February 2020).

4. Timæus IM, Moultrie TA. Teenage childbearing and educational attainment in South Africa. Stud Fam Plann 2015;46(2):143-160. https://doi.org/10.1111/j.1728-4465.2015.00021.x

5. Kaufman CE, de Wet T, Stadler J. Adolescent pregnancy and parenthood in South Africa. Stud Fam Plann 2001;32(2):147-160. https://doi.org/10.1111/j.1728-4465.2001.00147.x

6. Marteleto L, Lam D, Ranchhod V. Sexual behavior, pregnancy, and schooling among young people in urban South Africa. Stud Fam Plann 2008;39(4):351-368. https://doi.org/10.1111/j.17284465.2008.00180.x

7. Grant MJ, Hallman KK. Pregnancy-related school dropout and prior school performance in KwaZulu Natal, South Africa. Stud Fam Plann 2008;39(4):369-382. https://doi.org/10.1111/j.17284465.2008.00181.x 
8. Department of Education. Measures for the prevention and management of learner pregnancy 2007. https://www.gov.za/sites/default/files/gcis_document/201409/learnerpregnancy0.pdf (accessed 15 February 2020).

9. Panday S, Makiwane M, Ranchod C, Letsoala T. Teenage pregnancy in South Africa: With a specific focus on school-going learners. 2009. http://ecommons.hsrc.ac.za/handle/20.500.11910/4711 (accessed 15 February 2020).

10. Marteleto L, Lam D, Ranchhod V. Schooling and early childbearing in urban South Africa. PSC Research Report No. 06-610 2006. https://pdfs.semanticscholar.org/1f36/fc4c02263cb91a9ab8c79da7fc3f2f477b2d. pdf (accessed 15 February 2020).

11. Ardington C, Menendez A, Mutevedzi T. Early childbearing, human capital attainment and mortality risk: Evidence from a longitudinal demographic surveillance area in rural-KwaZulu-Natal, South Africa. Econ Dev Cult Change 2015;63(2):281-317. https://doi.org/10.1086/678983

12. Department of Education. DBE draft national policy on the prevention and management of learner pregnancy in schools. 2019. https://www.education.gov.za/Portals/0/Documents/Policies/Draft\%20 Pregnancy\%20Policy\%202018.pdf?ver=2018-06-26-142235-687 (accessed 15 February 2020).

13. Zweigenthal V, Strebel A, Hunter-Adams J. Adolescent girls perceptions of breastfeeding in two low-income periurban communities in South Africa. Health Care Women Int 2019;40(7-9):95-1011. https://doi.org/10.1080/07399332.2018.1549043

14. Wuestefeld M. Food and Nutrition Security. Meeting of the Minds: Nutrition Impact of Food Systems. Geneva: United Nations Standing Committee on Nutrition, 2013

15. Department of Health. Infant and young child feeding policy. 2013. https://health-e.org.za/wp content/ uploads/2013/09/IYCF_Policy_2013.pdf (accessed 15 February 2020).

16. Victora CG, Bahl R, Barros AJ, et al. Breastfeeding in the 21st century: Epidemiology, mechanisms, and lifelong effect. Lancet 2016;387(10017):475-490. https://doi.org/10.1016/s0140-6736(15)01024-7
17. Department of Education. Integrated school health policy. 2012. https://serve.mg.co.za/content/ documents/2017/06/14/integratedschoolhealthpolicydbeanddoh.pdf (accessed 15 February 2020).

18. Rasesemola RM, Matshoge GP, Ramukumba TS. Compliance to the integrated school health policy: Intersectoral and multisectoral collaboration. Curationis 2019;42(1):1-8. https://doi.org/10.4102/ Intersectoral and multise
curationis.v42il.1912

19. Lenkokile R, Hlongwane $P$, Clapper V. Implementation of the integrated school health policy in public primary schools in Region C, Gauteng Province. Afr J Pub Aff 2019;11(1):196-211

20. Dibakwane ST, Peu MD. Experiences of school health nurses regarding the provision of the schoo health service delivery in the Tshwane district. Afr J Prim Health Care Fam Med 2018;10(1):1-8. https://doi.org/10.4102/phcfm.v10i1.1807

21. Bhana D, Mcambi SJ. When schoolgirls become mothers: Reflections from a selected group of teenage girls in Durban. Perspect Med Educ 2013;31(1):11-19.

22. Stoner MCD, Pettifor A, Edwards JK, et al. The effect of school attendance and school dropout on incident HIV and HSV-2 among young women in rural South Africa enrolled in HPTN 068. AIDS 2007;31(15):2127-2134. https://doi.org/10.1097/qad.0000000000001584

23. Sullivan K, Clark J, Castrucci B, Samsel R, Fonseca V, Garcia I. Continuing education mitigates the negative consequences of adolescent childbearing. Matern Child Health J 2011;15(3):360-366. https:// negative consequences of adolesces

24. Freudenberg N, Ruglis J. Reframing school dropout as a public health issue. Prev Chronic Dis 2007;4(4):A107.

Accepted 14 April 2020. 